\title{
ARFAR: a person years at risk program
}

\author{
S F BARRY
}

From the National Radiological Protection Board, Chilton, Didcot, Oxon OX11 ORQ, UK

Cohort studies are often used to compare the health experience of groups of people. Several methods of analysis exist, such as the use of standardised or proportional mortality rates ${ }^{1}$ and the proportional hazards model. ${ }^{2}$ The calculation of a standardised mortality ratio (SMR) needs to take into account the effect of confounding factors, such as age and sex, which may affect mortality rates. The use of person years at risk to calculate an SMR is a means of controlling for these factors. ${ }^{3}$

Person years at risk are the number of years that the members of the study population contribute to the study. The person years may be divided into categories that contain only contributions from comparable individuals, thus controlling for the confounding effect. For example, since mortality rates vary with age the total person years at risk may be split into, say, five year age categories from age 20 to 70 . Mortality rates applicable to each age group may then be used to obtain an expected number of deaths in each age group and hence for all age groups. These rates might be taken from national figures, although this would not control for any "healthy worker" effect. ${ }^{4}$ The number of expected deaths may then be compared with the deaths observed in the study population. Similarly, it is possible to control for changes in mortality rates over time for a given age, by splitting the person years at risk according to calendar year. In the figure a subject enters the study at point $X$ and leaves at point $Y$, contributing at different times person years at risk to five age/calendar year categories. The appropriate age and calendar year specific death rates may be used to derive an expected number of deaths. Further divisions may also be made to allow for sex, race, or length of time in the study.

The calculation of person years at risk is a tedious task. Even splitting by as few as three factors can generate several hundred separate categories to use for the calculations. Several computer programs exist which enable the person years at risk to be calculated, among them MYCL, Man Years Computing Language. ${ }^{5}$ These programs allow the user to specify how

Accepted 3 December 1985 to split the person years at risk according to factors $\stackrel{\mathscr{O}}{\vec{\sigma}}$ such as age, calendar year, and length of time in $\stackrel{D}{D}$ study. In some occupational studies there may also be 3 available a quantitative measure of exposure to some $\vec{\omega}$ physical or chemical agent. Exposure of workers in $\dot{\infty}$ the nuclear industry to ionising radiation is an exam- of ple. Unlike the factors previously mentioned, such $N$ exposures do not necessarily increase at a constant rate. Whereas a person aged 30 in the year 1980 will $\rightarrow$ be aged 35 in the year 1985 , no such simple calculation may be made regarding radiation exposure. The program ARFAR (At Risk For Any Reason) has $\stackrel{\oplus}{\oplus}$ been written in Fortran to perform such calculations.

\section{Program}

ARFAR provides the facility to split person years at risk by age, calendar year, length of time in study, and a quantitative measure of exposure such as radiation $\stackrel{0}{\circ}$

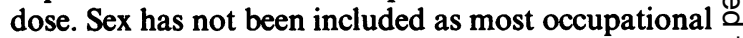
studies are performed on a single sex. Such an addi- $\overrightarrow{\vec{O}}$

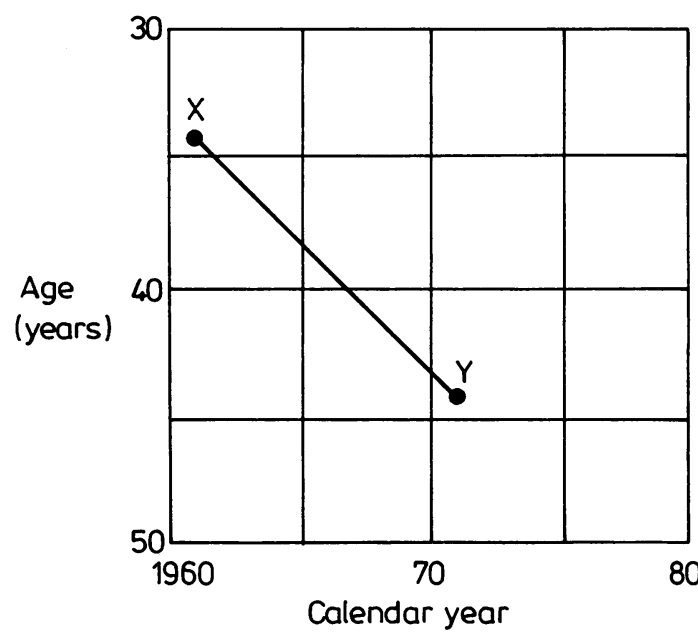

Diagram representing a subject entering a study at point $X$ and leaving at point $Y$. 
tional factor could be accommodated by making minor adjustments to the computer code, although this would necessarily increase the storage requirements. To run the program the user needs to specify the number of categories required for each stratifying factor and the end points of each category. Also required are the dates of birth, entry to and exit from the study for each person, plus a record of the dates and amounts of exposure. Although such records are often in the form of annual exposures, the dates in the exposure record may be at irregular intervals if desired. A known acute exposure could be handled by including exposures up to and just after the known event. The exposure received between two dates is considered to be received linearly. Using interpolation, ARFAR calculates the cumulative exposure at any time and hence calculates the day on which an individual changes exposure category. A latent period can also be specified such that the cumulative exposure at any time is considered to be equal to the exposure received up to, say, 10 years ago. In this way allowance may be made for the time between exposure and the resulting effect. For example, the minimal latent period for radiation induced cancers is often considered to be of the order of two to four years for leukaemia and $\mathbf{1 0}$ years for all other cancers. $^{6}$

The program produces tables of person years at risk and, if requested, tables of observed deaths from a specific cause. This output may then be used for subsequent analysis. ARFAR itself does not perform any tests but a further routine has been written which carries out a test for trend in mortality with exposure. ${ }^{7}$ This routine takes the output generated by ARFAR and calculates the test statistic.

$$
T=\sum_{\mathbf{i}, \mathbf{j}} \mathbf{d}_{\mathbf{j}}\left(\mathbf{O}_{\mathbf{i j}}-\mathbf{E}_{\mathbf{i j}}\right)
$$

Where $\mathrm{O}_{\mathrm{ij}}$ is the number of deaths observed in the job exposure category of the ith stratum, $E_{i j}$ is the number of deaths expected in the jth exposure category of the ith stratum conditional on the total number of deaths in that stratum, and assuming no effect due to the exposure, $d_{j}$ is the median of the recorded exposures falling in the jth exposure category in the entire data set. In the absence of any trend $E(T)=0$ and

$$
\operatorname{var}(T)=\sum_{i}\left[\sum_{j} d_{j}^{2} E_{i j}-\left\{\left(\sum_{j} d_{j} E_{i j}\right)^{2} /\left(\sum_{j} O_{i j}\right)\right\}\right]
$$

Source listings, documentation, and copies of the program are available. It is intended to incorporate constructive suggestions from interested users into later versions of this program. This work was partially supported by a CEC grant.

\section{References}

${ }^{1}$ Monson RR. Analysis of relative survival and proportional mortality. Comput Biomed Res 1974;7:325-32.

${ }^{2}$ Cox DR. Regression models and life tables. Journal of the Royal Statistical Society B 1972;34:187-220.

${ }^{3}$ Case RAM. Cohort analysis of mortality rates as a historical or narrative technique. Br J Prev Soc Med 1956;10:159-71.

4 Fox AJ, Collier PF. Low mortality rates in industrial cohort studies due to selection for work and survival in the industry. Br J Prev Soc Med 1976;30:225-30.

${ }^{5}$ Hill ID. Computing man years at risk. Br J Prev Soc Med 1972;26:132-4.

${ }^{6}$ Committee on the Biological Effects of Ionizing Radiation. The effects on populations of exposure to low levels of ionizing radiation. Washington: National Academy Press, 1980:193.

${ }^{7}$ Darby SC, Reissland JA. Low levels of ionising radiation and cancer-are we underestimating the risk? Journal of the Royal Statistical Society A 1981;144:298-331. 